\title{
Erratum to: Morrey Potentials and Harmonic Maps
}

\section{David R. Adams ${ }^{1}$, Jie Xiao ${ }^{2}$}

${ }^{1}$ Department of Mathematics, University of Kentucky, Lexington, KY 40506-0027, USA.

E-mail: dave.adams@uky.edu

2 Department of Mathematics and Statistics, Memorial University of Newfoundland, St. John's, NL A1C 5S7, Canada. E-mail: jxiao@mun.ca

Received: 21 April 2015 / Accepted: 13 May 2015

Published online: 11 July 2015 - ( ) Springer-Verlag Berlin Heidelberg 2015

Commun. Math. Phys. 308, 439-456 (2011)

There are two errors in our original paper:

- $\mathcal{I}_{\alpha} \mu$ in Theorem 2.4 should be replaced by $I_{\alpha} \mu$, and then $\approx$ in Theorem 2.4 (i) should be replaced by $\lesssim$.

- The estimate for the Wolff potential in the proof of Theorem 2.5 (plus Theorems 2.7 $\& 2.9$ ) is defective. So, the main parts of Theorems $2.5,2.7 \& 2.9$ should be replaced by

Theorem 0.1. Suppose $\mu$ and $f$ are a non-negative Radon measure and a non-negative function with support in a bounded domain $\Omega \subset \mathbb{R}^{N}$, respectively. Let

$$
\left\{\begin{array}{l}
1 \leq p<\infty \\
0<N-\alpha p<d \leq N ; \\
\|\mu\|_{d}<\infty ; \\
0<\|f\|_{L^{p, \lambda}}<\infty
\end{array}\right.
$$

(i) If $N \geq \lambda>\alpha p \& 1<q<\frac{p(d+\lambda-N)}{\lambda-\alpha p}$, then $\frac{\left(\int_{\Omega}\left|I_{\alpha} f\right|^{q} d \mu\right)^{\frac{1}{q}}}{\|f\|_{L^{p}, \lambda}} \lesssim\|\mu\|_{d}$.

(ii) If $N \geq \lambda>\alpha p \& 1<\gamma \leq q=\frac{p(d+\lambda-N)}{\lambda-p \alpha}$, then $\int_{\Omega}\left(\frac{I_{\alpha} f}{\|f\|_{L^{p, \lambda}}}\right)^{q}\left(\ln \left(1+\frac{I_{\alpha} f}{\|f\|_{L^{p, \lambda}}}\right)\right)^{-\gamma}$ $d \mu \lesssim\|\mu\|_{d}$

(iii) If $N \geq \lambda=\alpha p \& 0 \leq \beta \leq 1$, then there is a constant $c_{0}>0$ such that $\int_{\Omega} \exp \left(\frac{c I_{\alpha} f}{\|f\|_{L} p, \lambda}\right)^{\beta} d \mu \lesssim\|\mu\|_{d} \forall c \in\left[0, c_{0}\right]$.

JX is in part supported by NSERC of Canada and URP of Memorial University.

The online version of the original article can be found under doi:10.1007/s00220-011-1319-5. 
Proof. This follows from [3, Theorem 3.2].

The defectiveness has affected our paper [1]:

- $\quad[1$, Theorem 3.1] should be replaced by Theorem 0.1 .

- According to Theorem 0.1 , the condition $N \geq d>\lambda-\alpha p \quad \& \quad(\lambda, q) \in(0, N) \times$ $\left(0, \frac{d p}{\lambda-\alpha p}\right)$ in [1, Theorem 3.2] should be replaced by $N \geq d>N-\alpha p \quad \& \quad(\lambda, q) \in$ $(0, N) \times\left(0, \frac{(d+\lambda-N) p}{\lambda-\alpha p}\right)$. Thus, on [1, page 4811], $\frac{d p}{\lambda-\alpha p} ; \frac{d p}{\lambda-2 p} ; \frac{d p}{\lambda-(m+1) p}$ should be respectively replaced by $\frac{(d+\lambda-N) p}{\lambda-\alpha p} ; \frac{(d+\lambda-N) p}{\lambda-2 p} ; \frac{(d+\lambda-N) p}{\lambda-(m+1) p}$. Moreover, on [1, page 4812], $q<\frac{d p}{\lambda-\alpha p}$ should be replaced by $q<\frac{(d+\lambda-N) p}{\lambda-\alpha p}$.

- In accordance with Theorem 0.1(iii), $N \geq d>\lambda-\alpha p=0$ in [1, Theorem 3.6] should be replaced by $N \geq d>N-\alpha p>\lambda-\alpha p=0$.

Furthermore, this defectiveness has percolated down through our paper [2]:

- Since [2, Lemma 3.5] follows from [1, Theorem 3.1] which has been replaced by Theorem 0.1, [2, Lemma 3.5] should be replaced by the following result:

Let $\Omega \subset \mathbb{R}^{n}$ be a bounded domain, $0<\alpha, \lambda<n$ and $E \subseteq \Omega$.

(i) If $1<p<\lambda / \alpha, n-\alpha p<d \leq n$ and $1<q<p(d+\lambda-n) /(\lambda-\alpha p)$, then

$$
\Lambda_{d}^{(\infty)}(E) \lesssim\left(C_{\alpha}\left(E ; L^{p, \lambda}(\Omega)\right)\right)^{\frac{q}{p}}
$$

(ii) If $1<p=\lambda / \alpha, n-\alpha p<d \leq n$ and $0<q \leq 1$, then there is a constant $c>0$ such that

$$
\Lambda_{d}^{(\infty)}(E) \lesssim \exp \left(-c\left(C_{\alpha}\left(E ; L^{p, \lambda}(\Omega)\right)\right)^{\frac{q}{p}}\right)
$$

- According to Theorem 0.1, the Hausdorff dimension estimates in [2, Theorem 2.1]; [2, Theorem 3.6(i)]; [2, Theorem 3.6(ii)], should be respectively replaced by:

$$
\left\{\begin{array}{l}
\operatorname{dim}_{H}\left(\Sigma_{\hat{p}}(u, \Omega)\right) \leq n-m p \\
\operatorname{dim}_{H}\left(\Sigma_{1}\left(I_{\alpha} f, \Omega\right)\right) \leq n-\alpha p \\
\operatorname{dim}_{H}\left(\Sigma_{1}\left(I_{\alpha} f, \Omega\right)\right) \leq n-\alpha p
\end{array}\right.
$$

- The Hausdorff dimension of any local singular set in [2, Theorem 2.2] is at most $n-2$. This follows from Theorem 0.1(iii).

- On [2, page 1264], $d>\mu-\alpha p ; 0<q<\frac{p(\mu-\alpha p)}{d} ; \lambda-\alpha p$ should be replaced by $d>n-\alpha p ; 1<q<\frac{p(d+\mu-n)}{\mu-\alpha p} ; n-\alpha p$, respectively.

- On [2, page 1265], $d>\mu-\alpha p>\lambda-\alpha p \quad \& \quad \operatorname{dim}_{H}\left(\mathrm{~T}\left(I_{\alpha} f, \Omega\right)\right) \leq \lambda-\alpha p$ should be replaced by $d>n-\alpha p \quad \& \quad \operatorname{dim}_{H}\left(\mathrm{~T}\left(I_{\alpha} f, \Omega\right)\right) \leq n-\alpha p$, respectively.

- On [2, page 1266], $\operatorname{dim}_{H}\left(\Sigma_{\hat{p}}(u, \Omega)\right) \leq n p / q$ should be replaced by $\operatorname{dim}_{H}\left(\Sigma_{\hat{p}}(u, \Omega)\right)$ $\leq n-m p$.

- On [2, page 1268], $d>\epsilon+n p / q \& \operatorname{dim}_{H}\left(\Sigma_{p}(g, \Omega)\right) \leq n p / q$ should be replaced by $d>\epsilon+n-m p \& \operatorname{dim}_{H}\left(\Sigma_{p}(g, \Omega)\right) \leq n-m p$, respectively. 
- On [2, pages 1269-1270],

$$
\operatorname{dim}_{H}\left(\Sigma_{2}(u, B)\right)=0 ; \operatorname{dim}_{H}\left(\Sigma_{2}\left(v, \mathbb{B}^{n}\right)\right)=0 ; \operatorname{dim}_{H}\left(\Sigma_{2}(u, \Omega)\right)=0
$$

should be replaced respectively by [due to Theorem 0.1(iii)]

$\operatorname{dim}_{H}\left(\Sigma_{2}(u, B)\right) \leq n-2 ; \operatorname{dim}_{H}\left(\Sigma_{2}\left(v, \mathbb{B}^{n}\right)\right) \leq n-2 ; \operatorname{dim}_{H}\left(\Sigma_{2}(u, \Omega)\right) \leq n-2$.

Consequently, "Therefore, $\Sigma_{2}(u, \Omega)$ consists of at most isolated points" on [2, page 1270] and the second part of [2, page 1260: Theorem 2.2] are uncertain under $n \geq 3$.

We would like to thank Xavier Cabré who questioned the Wolff potential estimate for verifying Theorem 2.5, which led us to the discovery of the errors in the original paper.

\section{References}

1. Adams, D.R., Xiao, J.: Regularity of Morrey commutators. Trans. Am. Math. Soc. 364, 4801-4818 (2012)

2. Adams, D.R., Xiao, J.: Singularities of nonlinear elliptic systems. Commun. Partial Differ. Equ. 38, 12561273 (2013)

3. Adams, D.R., Xiao, J.: Restrictions of Riesz-Morrey potentials (2015) (preprint) 